\title{
The present theoretical error on the Bhabha scattering cross section in the luminometry region at LEP
}

\author{
A. Arbuzovi ${ }^{1}$, M. Bigi ${ }^{2}$, H. Burkhardt ${ }^{3}$, M. Cacciari ${ }^{4}$, M. Caffo ${ }^{5}$, H. Czyż $\dot{z}^{6}$, M. Dallavalle ${ }^{7}$, \\ J. H. Field ${ }^{8}$, F. Filthaut ${ }^{9}$, S. Jadach ${ }^{10}$, F. Jegerlehner ${ }^{11}$, E. Kuraev ${ }^{12}$, G. Montagna ${ }^{13}$, \\ O. Nicrosini ${ }^{14}$, F. Piccinini ${ }^{15}$, B. Pietrzyk ${ }^{16}$, W. Płaczek ${ }^{17}$, E. Remiddi ${ }^{18}$, M. Skrzypek ${ }^{19}$, \\ L. Trentadue ${ }^{20}$, B. F. L. Ward ${ }^{21}$, Z. Wa̧s ${ }^{22}$
}

\footnotetext{
${ }^{1}$ BLTP, JINR, Dubna, 141980, Russia, ARBUZOV@THSUN1.JINR.DUBNA.SU; work supported in part by the Royal Swedish Academy of Sciences (ICFPM grant)

${ }^{2}$ Dipartimento di Fisica Sperimentale, Università di Torino and INFN, Via P. Giuria 1, I-10125 Turin, Italy, MBIGI@VXCERN.CERN.CH

${ }^{3}$ CERN, SL Division, CH-1211 Geneva 23, Switzerland, HBU@CERNVM.CERN.CH

${ }^{4}$ DESY, Hamburg, Germany, CACCIARI@DESY.DE; DESY postdoc

${ }^{5}$ INFN and Dipartimento di Fisica dell'Università, Bologna, Italy, CAFFO@BO.INFN.IT

${ }^{6}$ Institute of Physics, University of Silesia, Katowice, Poland; INFN and Dipartimento di Fisica dell'Università, Bologna, Italy, CZYZ@BO.INFN.IT; work supported in part by USA-Poland Maria Sklodowska-Curie Joint Fund II, grant MEN/NSF-93-145, and Polish Committee for Scientific Research, grant no. 2P03B17708

${ }^{7}$ INFN and Dipartimento di Fisica dell'Università, Bologna, Italy, DVM@CERNVM.CERN.CH

${ }^{8}$ University of Geneva, Switzerland, JFIELD@CERNVM.CERN.CH

${ }^{9}$ Carnegie Mellon University, Pittsburgh, PA 15213, USA, FILTHAUT@HPL3.CERN.CH

${ }^{10}$ Institute of Nuclear Physics, ul. Kawiory 26A, 30-055 Kraków, Poland, and CERN, TH Division, CH-1211 Geneva 23, Switzerland, JADACH@CERNVM.CERN.CH; work supported in part by Polish Committee for Scientific Research, grant no. 2P30225206, US DoE contract DE-FG05-91ER40627, and IN2P3 French-Polish Collaboration through LAPP Annecy

${ }^{11}$ DESY Zeuthen, Germany and ETH Zurich, Switzerland, FJEGER@IFH.DE

${ }^{12}$ BLTP, Dubna, 141980, Russia, KURAEV@THEOR.JINRC.DUBNA.SU

${ }^{13}$ Dipartimento di Fisica Nucleare e Teorica and INFN, Pavia, Italy, MONTAGNA@PAVIA.PV.INFN.IT

${ }^{14}$ INFN and Dipartimento di Fisica Nucleare e Teorica dell'Università, Pavia, Italy, NICROSINI@PAVIA.PV.INFN.IT ${ }^{15}$ INFN, Pavia, Italy, PICCININI@PAVIA.PV.INFN.IT

${ }^{16}$ LAPP, IN2P3-CNRS, F-74941 Annecy-le-Vieux Cedex, France, PIETRZYK@LAPP.IN2P3.FR

${ }^{17}$ Department of Physics and Astronomy, The University of Tennessee, Knoxville, Tennessee 37996-1200, USA (On leave of absence from Institute of Computer Science, Jagellonian University, ul. Reymonta 4, Kraków, Poland), PLACZEK@HEPHP02.PHYS.UTK.EDU; work supported in part by US DoE contract DE-FG0591ER40627

${ }^{18}$ INFN and Dipartimento di Fisica dell'Università, Bologna, Italy, REMIDDI@BO.INFN.IT

${ }^{19}$ Institute of Nuclear Physics, ul. Kawiory 26A, 30-055 Kraków, Poland, SKRZYPEK@HPJMIADY.IFJ.EDU.PL; work supported in part by Polish Committee for Scientific Research, grant no. 2P30225206, European Commission contract ERBCIPDCT940016, and IN2P3 French-Polish Collaboration through LAPP Annecy

${ }^{20}$ Dipartimento di Fisica, Università di Parma, and INFN, Gruppo Collegato di Parma, Sezione di Milano, Italy, TRENTA@CERNVM.CERN.CH, TRENTA@VXCERN.CERN.CH, TRENTADUE@ROMA2.INFN.IT

${ }^{21}$ Department of Physics and Astronomy, The University of Tennessee, Knoxville, Tennessee 37996-1200, USA, and SLAC, Stanford University, Stanford, California 94309, USA, BFLW@SLACVM.SLAC.STANFORD.EDU; work supported in part by US DoE contract DE-FG05-91ER40627

${ }^{22}$ CERN, TH Division, CH-1211 Geneva 23, Switzerland, and Institute of Nuclear Physics, ul. Kawiory 26A, 30-055 Kraków, Poland, WASM@CERNVM.CERN.CH; work supported in part by Polish Committee for Scientific Research, grant no. 2P30225206
} 


\begin{abstract}
The results concerning the theoretical evaluation of the small-angle Bhabha Scattering cross section obtained during the Workshop on Physics at LEP2 (CERN, Geneva, Switzerland, 1995) by the Working Group "Event Generators for Bhabha Scattering" are summarized. The estimate of the theoretical error on the cross section in the luminometry region is updated.
\end{abstract}

Submitted to Physics Letters B

May 6, 1996 
During 1995, within the Workshop on Physics at LEP2, a Working Group on "Event Generators for Bhabha Scattering" was convened. The main tasks of the Working Group were

- to make an inventory of all the available Monte Carlo (MC) event generators, developed by independent collaborations, for small-angle (SABH) and large-angle (LABH) Bhabha processes at LEP1 and LEP2;

- to improve our understanding of their theoretical uncertainties by means of systematic comparisons of MC's between themselves and with non-MC approaches.

The main emphasis was put on SABH processes, because of the pressing need to match the theoretical precision of the calculations with the much improved experimental accuracy $(\leq 0.1 \%)$ of the luminosity measurement. In particular, the main achievement of the program outlined, which is the result of a combined effort by several collaborations addressing several theoretical and experimental issues, was the reduction of the theoretical error on the SABH cross section from $0.16 \%$ to $0.11 \%$ for typical event selections (ES) at LEP1, and a first estimate of the theoretical error on the SABH cross section at LEP2.

The aim of the present short note is to summarize the strategies adopted in order to achieve the goal stated above, and to officially state the conclusions drawn by the Working Group. For any details concerning experimental aspects, or theoretical issues, as well as descriptions of the codes involved, the reader is referred to [1], where the proper references to all the individual contributions can be found.

The various components of the theoretical error on the SABH cross section are quoted in Tab. 1, where a summary of the past and present situation at LEP1 together with the present estimate valid for LEP2 is given. The errors in the table are understood to be attributed to the cross section for any typical (asymmetric) ES, for a LEP1 experiment in the angular range $1^{\circ}-3^{\circ}$, calculated using BHLUMI 4.03. In the case of LEP2, the estimate extends to the angular range $3^{\circ}-6^{\circ}$, and also to a possible narrower angular range (say $4^{\circ}-6^{\circ}$ ) that may be necessary due to the effect of synchrotron radiation masks in the experiments. The entries include combined technical and physical precision.

\begin{tabular}{||l|l|l|l||}
\hline \hline & \multicolumn{2}{|c|}{ LEP1 } & LEP2 \\
\hline Type of correction/error & Past & Present & Present \\
\hline (a) Missing photonic $\mathcal{O}\left(\alpha^{2} L\right)$ & $0.15 \%$ & $0.10 \%$ & $0.20 \%$ \\
(b) Missing photonic $\mathcal{O}\left(\alpha^{3} L^{3}\right)$ & $0.008 \%$ & $0.015 \%$ & $0.03 \%$ \\
(c) Vacuum polarization & $0.05 \%$ & $0.04 \%$ & $0.10 \%$ \\
(d) Light pairs & $0.01 \%$ & $0.03 \%$ & $0.05 \%$ \\
(e) Z-exchange & $0.03 \%$ & $0.015 \%$ & $0.0 \%$ \\
\hline Total & $0.16 \%$ & $0.11 \%$ & $0.25 \%$ \\
\hline \hline
\end{tabular}

Table 1: Summary of the total (physical+technical) theoretical uncertainty for a typical calorimetric detector. For LEP1, the above estimate is valid for the angular range within $1^{\circ}-3^{\circ}$, and for LEP2 it covers energies up to $176 \mathrm{GeV}$, and angular range within $1^{\circ}-3^{\circ}$ and $3^{\circ}-6^{\circ}$ (see the text for further comments). 
As can be seen in the table, at the present stage the theoretical error is still dominated by the error on photonic corrections, quoted in entries (a) and (b), and in particular by the one due to missing $\mathcal{O}\left(\alpha^{2} L\right)$, where $L$ is the usual collinear logarithm $L=\ln \left(-t / m^{2}\right)$, of entry (a). Since the error of entry (a) is by far dominant with respect to all the other ones, it is worth devoting some space to describe how it has been estimated, namely by adopting the following procedure:

- consideration of only the photonic corrections to the dominant part of the SABH cross section, namely the one due to $t$-channel photon exchange;

- to define four families of ES's, starting from the simplest one, BARE1, in which cuts are applied only to the "bare" final fermions, and going, through CALO1 and CALO2, implementing more and more complex clustering algorithms, to SICAL2, which is very similar to a "real" experimental ES; since photonic corrections are very sensitive to the details of the ES, defining these four ES's allows to span in detail the photonic phase space; even if the ES BARE1 is far from realistic, the presently available analytical calculation including the complete set of $\mathcal{O}\left(\alpha^{2} L\right)$ corrections refers to such an ES, and so provides a very important cross-check of the MC programs;

- to run all the available codes for all the ES's, varying inside any ES the threshold requirements for the final state fermions/clusters;

- to perform a test concerning the technical precision, namely comparing the exact up to $\mathcal{O}(\alpha)$ cross sections; agreement at the level of a few $10^{-4}$ relative deviation has been achieved (for brevity not shown here);

- finally, to compare the results of the codes including the full higher-order photonic corrections in each case, for all the situations explored (see Fig. 11).

The result of this procedure allowed the definition of "one-per-mill regions", referring to realistic threshold cuts, within which most of the predictions lie. Moreover, for those cases for which the predictions do not lie within the "one-per-mill regions", the reasons for the deviations involved have been carefully investigated and eventually understood. An analogous procedure has been followed after the inclusion of all the relevant radiative corrections (vacuum polarization, $Z$ contributions and so on), and extending the comparisons also to asymmetric ES's, leading to the results shown as an example in Fig. 2. 1 A similar analysis has also been performed for the first time in situations which will be typical at the LEP2 experiments. The conclusion drawn at the end of all these comparisons is that now the theoretical uncertainty due to uncontrolled $\mathcal{O}\left(\alpha^{2} L\right)$ corrections is reduced from $0.15 \%$ to $0.10 \%$ for the LEP1 situation, and estimated to be $0.20 \%$ at LEP2. As far as entry (b), the "missing photonic $\mathcal{O}\left(\alpha^{\ni} \mathcal{L}^{\ni}\right)$ " uncertainty, is concerned, new estimates of the effect have resulted in a more conservative theoretical error, namely $0.015 \%$ to be compared with the old estimate of $0.008 \%$.

*The results by BHAGEN95 shown here are slightly changed with respect to the ones quoted in [1], due to a bug-fixing.

${ }^{\dagger}$ In the semi-analitical program NLLBHA in the BARE1 set-up of Fig. 1 all the corrections of $\mathcal{O}\left(\alpha^{\in} \mathcal{L}\right)$ and of $\mathcal{O}\left(\alpha^{\ni} \mathcal{L}^{\ni}\right)$ are contained. 
As far as all the other entries in Tab. 11 are concerned, namely entries (c), the "vacuum polarization" uncertainty, (d), the "light pairs" uncertainty, and (e), the " $Z$-exchange" uncertainty, two of them, (c) and (e), are reduced with respect to the previous situation thanks to several new fits of the hadronic contribution to the vacuum polarization, and some additional original work on the $Z$-exchange contribution done during the workshop. New estimates, both Monte Carlo and analytical, of the light pairs contribution, (d), featuring more complete calculations done during the workshop, have resulted in a more conservative estimate of the pairs effect uncertainty of $0.03 \%$; 用 if it would be necessary, this effect can be included in the BHLUMI event simulation itself using an extension of the respective YFS exponentiation to soft pairs radiation via methods already represented in [1] and references therein.

In conclusion, the total (physical + technical) theoretical uncertainty on the SABH cross section for a typical calorimetric detector is at present $0.11 \%$ at LEP1 and $0.25 \%$ at LEP2.

Acknowledgements - M. Cacciari thanks the Fondazione A. Della Riccia and the Università di Pavia for supporting his staying at DESY when this work was performed. H. Czyż thanks OPAL collaboration for the support and kind hospitality during completion of this work. B.F.L. Ward thanks Profs. G. Veneziano and G. Altarelli and Prof. D. Schlatter for the support and kind hospitality of the CERN TH Division and of the ALEPH Collaboration, respectively, while this work was completed.

\section{References}

[1] "Event Generators for Bhabha Scattering", by S. Jadach (convener), O. Nicrosini (convener), H. Anlauf, A. Arbuzov, M. Bigi, H. Burkhardt, M. Cacciari, M. Caffo, H. Czyż, M. Dallavalle, J. H. Field, F. Filthaut, F. Jegerlehner, E. Kuraev, G. Montagna, T. Ohl, F. Piccinini, B. Pietrzyk, W. Płaczek, E. Remiddi, M. Skrzypek, L. Trentadue, B. F. L. Ward, Z. Wạs, in "Physics at LEP2", G. Altarelli, T. Sjöstrand and F. Zwirner eds., CERN 96-01, vol. 2, p. 229; e-Print Archive: hep-ph/9602393.

\footnotetext{
${ }^{\ddagger}$ Table 20 in 11 contains a copying error in its light pairs row for the Past LEP1 entry, where $0.04 \%$ should be $0.01 \%$, as we show it here.
} 

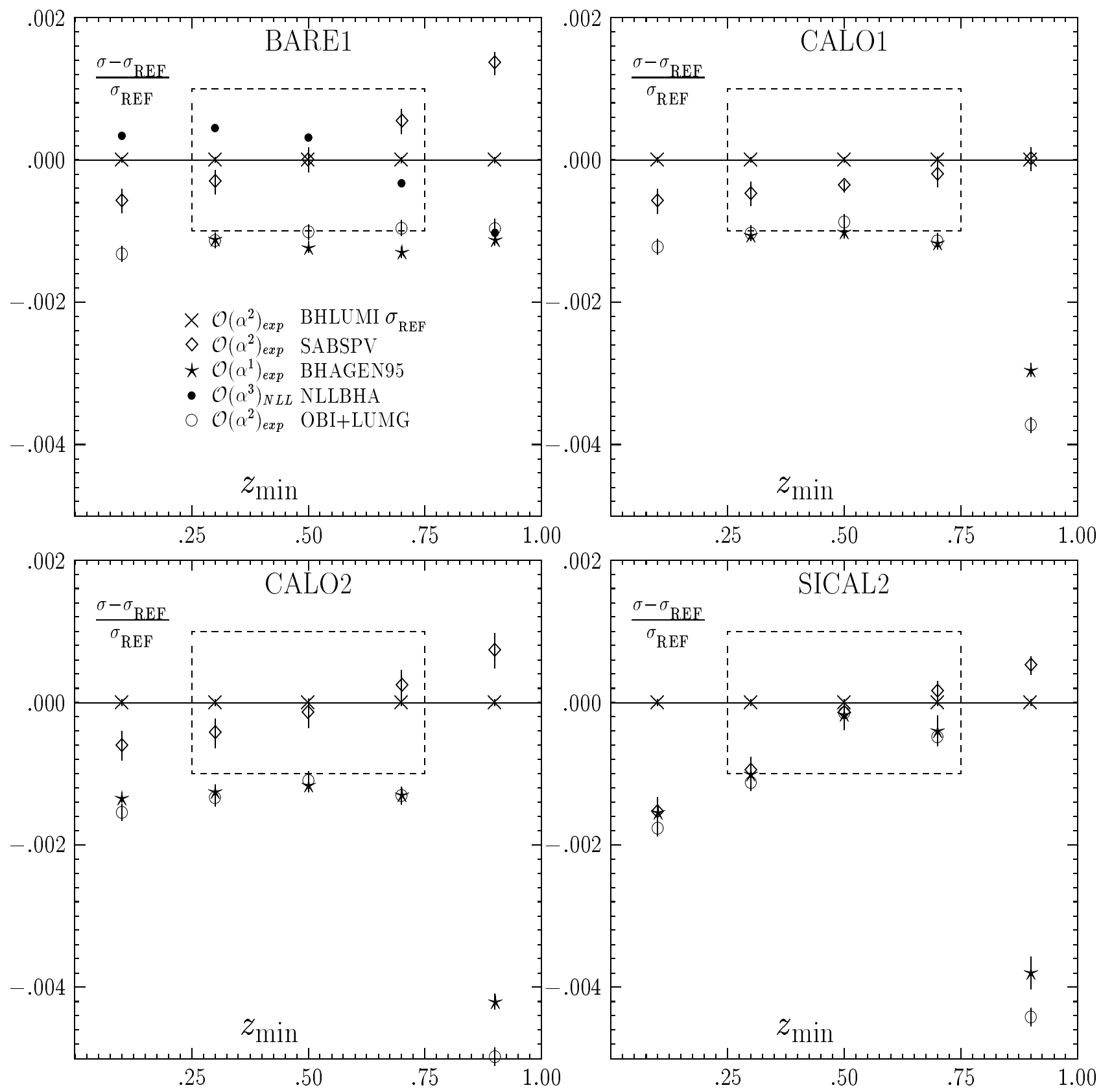

Figure 1: Monte Carlo results for the symmetric Wide-Wide ES's BARE1, CALO1, CALO2 and SICAL2, for matrix elements beyond first order. Z exchange, up-down interference and vacuum polarization are switched off. The center of mass energy is $\sqrt{s}=92.3 \mathrm{GeV} . z_{\min }$ is the cut condition on the final-state energies, defined as $E_{+} E_{-} / E^{2} \geq z_{m i n}, E_{-,+}$being the final-state energy of the bare electron and positron, respectively. The fiducial angular range is $0.024-0.058 \mathrm{rad}$ (for more details on the ES, the reader is referred to [1]). In the plot, the $\mathcal{O}\left(\alpha^{2}\right)_{\exp }^{Y F S}$ cross section from BHLUMI 4.03 is used as a reference cross section. 

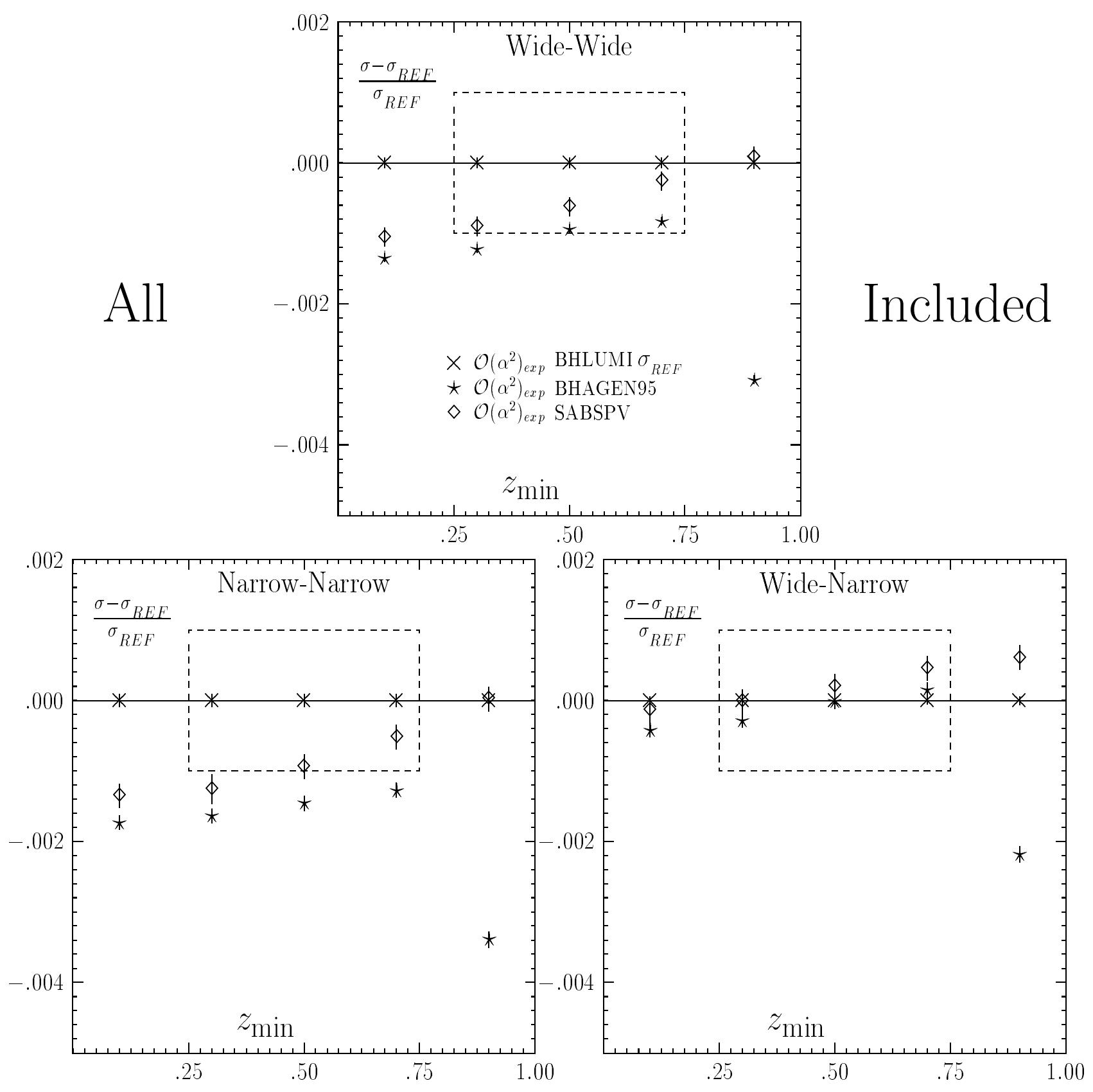

Figure 2: Monte Carlo results for various symmetric/asymmetric versions of the CALO2 ES, for matrix elements beyond first order. Z exchange, up-down interference and vacuum polarization are switched ON. The center of mass energy is $\sqrt{s}=92.3 \mathrm{GeV} . z_{\min }$ is the cut condition on the final-state energies, defined as $E_{+} E_{-} / E^{2} \geq z_{\min }, E_{-,+}$being the final-state energy of the electron and positron clusters, respectively. The fiducial angular range is 0.024-0.058 rad (for more details on the ES, the reader is referred to [1]). In the plot, the $\mathcal{O}\left(\alpha^{2}\right)_{\exp }^{Y F S}$ cross section from BHLUMI 4.03 is used as a reference cross section. 